\title{
Determination of Aquaponic Water Macronutrient Concentrations Based on Lactuca Sativa Leaf Photosynthetic Signatures using Hybrid Gravitational Search and Recurrent Neural Network
}

\author{
Ronnie CONCEPCION II ${ }^{1, *}$, EImer DADIOS ${ }^{2}$, Joel CUELLO ${ }^{3}$, \\ Argel BANDALA ${ }^{1}$, Edwin SYBINGCO ${ }^{1}$ and Ryan Rhay VICERRA ${ }^{2}$ \\ ${ }^{I}$ Department of Electronics and Communications Engineering, De La Salle University, Manila, Philippines \\ ${ }^{2}$ Department of Manufacturing Engineering and Management, De La Salle University, Manila, Philippines \\ ${ }^{3}$ Department of Agricultural and Biosystems Engineering, University of Arizona, Tucson, USA
}

('Corresponding author's e-mail: ronnie_concepcionii@dlsu.edu.ph)

Received: 25 January 2021, Revised: 21 April 2021, Accepted: 28 April 2021

\begin{abstract}
Crop quality depends dominantly on the nutrients present in its growth media. For precision farming, fertigation is a challenge, especially when dealing with economical and efficiency factors. In this study, the aquaponic pond water macronutrient prediction model (wNPK) was developed based on leaf photosynthetic signature predictors. Aquaphotomics was preliminarily used for correlating physical limnological properties with nitrate, phosphate, potassium concentrations, and the leaf signatures. Using a digital camera, 18 spectro-textural-morphological features were extracted. Neighborhood component analysis (NCA) and ReliefF algorithms selected the spectral components blue, a*, and red minus luma as the most significant as supported by principal component analysis, resulting in low computational cost. A Gravitational Search Algorithm (GSA) was employed to optimize the recurrent neural network (RNN) architecture resulting in higher sensitivity. The hybrid NCA-ReliefF-GSA-RNN (wNPK) predicted NPK with 93.61, 84.03, and 91.39\% accuracy, respectively, besting out other configured feature-based machine learning models. Using wNPK, it was confirmed that potassium helped in accelerating seed germination and nitrogen in promoting chlorophyll intensification, especially on the $6^{\text {th }}$ week after sowing. Phosphate and potassium were the energy and health elements that were consumed in a larger amount at the end of the head development stage. wNPK rules out that macronutrient concentration have a direct resemblance to crop leaf signatures; thus, a leaf is a good indicator of the water quality. The results pointed out that the use of a single camera to measure both water macronutrient concentrations and crop signature at the same time is an innovative, efficient, and economical approach for precision farming.
\end{abstract}

Keywords: Aquaponics, Aquaphotomics, Lettuce, Precision farming, Water nutrient concentration

\section{Introduction}

In agriculture, water is the source of fundamental nutrients essential for plant growth. It is an inorganic and almost colorless compound in its purest form at room temperature that contains a substantial amount of macronutrients and micronutrients. For plant nutrient nutrition, macronutrient is defined as the collection of elements that are required in a large amount to induce plant growth. It consists of nitrogen $(\mathrm{N})$, phosphorus $(\mathrm{P})$, potassium $(\mathrm{K})$, hydrogen $(\mathrm{H})$, oxygen $(\mathrm{O})$, carbon $(\mathrm{C})$, sulfur $(\mathrm{S})$, calcium $(\mathrm{Ca})$, and magnesium $(\mathrm{Mg})$. Micronutrients, on the other hand, are an indispensable cluster of nutrients that are acquired in a lesser amount than the macronutrients. It mainly consists of iron (Fe), copper $(\mathrm{Cu})$, zinc $(\mathrm{Zn})$, manganese $(\mathrm{Mn})$, molybdenum $(\mathrm{Mo})$, chlorine $(\mathrm{Cl})$, nickel $(\mathrm{Ni})$, and boron $(\mathrm{B})$. 
http://wjst.wu.ac.th

These nutrients are naturally fostered by soil fertility where the water system is exposed. Soil nutrients have been quantified using the standard approach of near-infrared laser absorption [1]. Both macro-and micronutrients are obtained by aquatic plants in the form of ions. For any cases that there is soil nutrient infertility, the possibility of water nutrient concentration is expected to be low especially when there are no aquatic animals present in the system that could provide additional nutrients. In the case of aquaponics, a revolutionary scheme of soilless agriculture that cultivates and produces fish and crops using water as a key growth medium, water is the primary promoter of plant growth. Thus, plant leaf properties have a direct resemblance to the existing water nutrient concentration [2].

Carbon, hydrogen, and oxygen are abundant in open space and can be easily derived from water and air. However, nitrogen, phosphorus, and potassium (NPK), as the primary macronutrients, are vulnerable to imbalance and shortage depending on abiotic factors including temperature and biotic factors such as microorganisms and animals. The nitrogen cycle is exhibited in this cultivation setup as fish effluents are mixed onto the water system where Nitrosomonas decomposes it into nitrites $\left(\mathrm{NO}_{2}{ }^{-}\right)$and Nitrobacter decomposes nitrites into nitrates $\left(\mathrm{NO}_{3}{ }^{-}\right)$which is considered as vital plant food. Hence, nitrogen constitutes half of the dry matter of photosystems and protoplasm of plants. Phosphorus is essential for the primary and secondary growth of roots, and potassium is responsible for water movement and flowering. Increasing amounts of nitrate, phosphate, and potassium are lethal to fish and plants in an aquaponic environment due to the existence of eutrophication [3]. Deprivation of each of these primary macronutrients is reflective to plant architecture. The lake hydrochemical data, namely, phosphate and nitrate concentrations were quantified using the Winkler method and spectrophotometry [4]. The total phosphorus dissolved in lake water was determined using the molybdenum blue colorimetric method [5].

A pond is a shallow and isolated water ecosystem that is topographically smaller than a lake. Pond biodiversity varies depending on the living organisms existing in it. Most frequently, artificial ponds are expected to be used in controlled environment agriculture (CEA) [6]. Its floor and walls can be constructed using plastic tarpaulins or cement. However, in this setup, the interaction of soil microorganisms to water nutrient enhancement is limited. In the Philippines, artificial ponds are still observed to have a certain mixture of soil components [7]. By default, artificial pond water cannot provide sufficient nutrients for plant intake without fish effluents or other suppliers of nutrients. Even the feed composition for fishes impacts the nutrient concentration of water [8]. Hence, proper nutrient and irrigation systems must be incorporated in any type of cultivation system to have higher yields. Nutrient management systems must be based on the nutrient requirement of crops to ensure on-time production [9].

The automated irrigation and fertigation system employs the concept of precision agriculture that controls the manages the amount of nutrient-rich water to flow through crop grow beds [10]. NPK concentrations were determined using sensors that are connected to a microcontroller and relay control circuit [11]. These sensors can even reach a $60 \mathrm{~s}$ response time [12]. It is usually done using weather and limnological sensors. In light of the efficient and optimal application of fertilizer, nitrogen, and phosphorus concentrations for tobacco farming were quantified using flame spectrophotometry [13]. Weather-based determination of water nutrients is characterized by the amount of precipitation, environment humidity and temperature, wind speed, and solar irradiation [14]. The soil and water assessment tool (SWAT) was developed based on generalized likelihood uncertainty estimation (GLUE) and implemented in 3 open-field water reservoirs [14]. An aquaponic setup of pakchoi (Brassica rapa L.) and tilapia (Oreochromis niloticus) was constructed, and the ammonia, nitrate and nitrite concentrations were measured using water sampling and processed using ultraviolet-visible (UV-Vis) spectrophotometer in the 190 to $1100 \mathrm{~nm}$ spectrum [15]. In a recirculating aquaculture system (RAS), sulfate strengthens the hydrogen sulfide $\left(\mathrm{H}_{2} \mathrm{~S}\right)$ concentration in water that is dangerous to all microorganisms and fishes living in it. Hence, a method of determining sulfide concentration was developed using methylene blue [16,17]. The carbon and hydrogen concentrations on an artificial aquaponic pond were measured using UV-Vis spectrophotometry in the range of 100 to $1000 \mathrm{~nm}$ and multigene symbolic regression genetic programming (MSRGP) [18] and phosphate concentration are predicted using an adaptive neuro-fuzzy inference system (ANFIS) [19]. The nitrate concentration in a water reservoir was monitored using the cadmium-reduction method through the cadmium column system [20]. Moreover, an automated 
integrated syringe-pump-based environmental-water analyzer has been proven successful in analyzing nitrate and nitrite concentrations in an estuarine system [21]. Dissolved carbon dioxide $\left(\mathrm{CO}_{2}\right)$ concentration in a lake was measured using satellite estimation [22]. NPK concentration has been measured using color sensor TCS3200 [23].

Despite the abovementioned advances in nutrient detection and monitoring in agriculture, leaf-based detection of water nutrients is not yet done. The techniques currently used worldwide are more on spectrophotometry and aquatic chemical sensors which are expensive. The prior technique cannot be employed in real-time monitoring of water nutrient concentrations which raises a problem in the very active water system in an aquaponic environment. As of this writing, no published studies have correlated crop leaf properties to water nutrient concentration in a soilless environment as a basis for an automated nutrient management system.

In this study, a feature-based machine learning model with lettuce leaf canopy photosynthetic signatures as inputs was developed to predict the nutrient concentration present on the aquaponic pond water where plant roots are biologically connected. The developed model is named wNPK which stands for water nitrate, phosphate, and potassium concentrations that is technically the output of this AI model. A digital RGB camera is used to capture leaf canopy images. Electronic temperature, $\mathrm{pH}$, and electrical conductivity sensors were used to acquire limnological data for aquaphotomic analysis in correlating it with ultraviolet-visible light (UV-Vis) spectrophotometrically generated nitrate, phosphate, and potassium concentrations. A Gravitational Search Algorithm was used to optimizing the selected feature-based machine learning model to yield acceptable prediction accuracy and sensitivity. Spectro-texturalmorphological leaf signatures were considered because they can be extracted using non-destructive imaging which is ideal when cultivating a limited population of crops. It is also better than measuring manually the fresh weights of crops as not to disturb their natural growth condition. This developed lowcost approach in detecting water nutrient concentration employing a consumer-grade camera is an essential innovation in precision farming. It is part of the vision-based lettuce phenotype (VIPHLET) model that is implemented in a lettuce smart farm.

\section{Materials and methods}

The model for predicting aquaponic water macronutrient concentrations requires both water and lettuce leaf samples as inputs during training (Figure 1). The process involves physical limnological properties acquisition through electronic sensors, aquaphotomics modeling, leaf canopy feature extraction, and machine learning modeling (Figure 1). However, the final model outputs nitrate, phosphate, and potassium concentrations by just setting the captured lettuce phenotypic image as input.

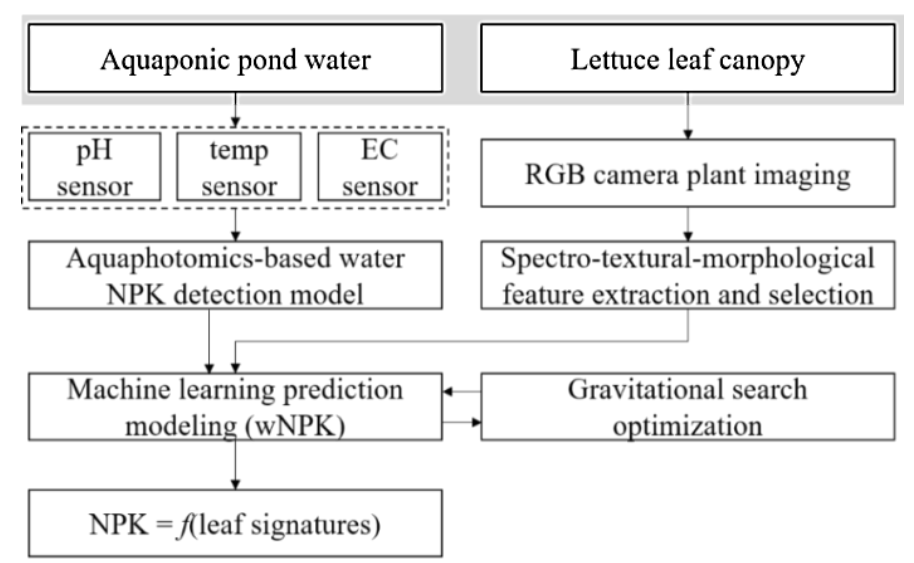

Figure 1 General architecture of the development of aquaponic water macronutrient concentrations prediction model based on lettuce leaf signatures. 


\section{Plant material and controlled environment cultivation}

Loose-leaf green lettuce, or Lactuca sativa Altima (Ramgo International Corporation, Philippines), is the chosen cultivar to be grown inside a controlled aquaponic chamber for a 42-day complete plant life cycle from August to September $2020\left(14.5181^{\circ} \mathrm{N}, 121.2390^{\circ} \mathrm{E}\right)$. To provide sufficient nutrients on the water for plant intake, an aquaculture tilapia and carps (Bureau of Fisheries and Aquatic Resources, Philippines) was placed on an artificial freshwater pond that is connected to the 2-layer nutrient film technique (NFT) plant growth chamber through a water pump. NFT vessel is made up of 3-inch foodgrade polyvinyl chloride (PVC) pipes configured in serpentine vertical farming configuration (Figure 2). Green rockwool set at the bottom of a 2-inch plastic cup is the employed growth media for the crop. The top layer of the growth rack houses the red and blue photosynthetic light source using LED strips. The lower rack is equipped with a T8 LED full-spectrum light source. The photoperiod is set to 18 light $\mathrm{h}$ (6:00 a.m. to 10:00 p.m.) and 6 dark h (10:00 p.m. to 6:00 a.m.). It is automatically enabled by a lighting system developed using Arduino UNO and relay driver. For each layer, 20 lettuces were directly germinated on green rockwools with a planting distance of 6 inches on each side. Aside from weekly aquaculture tank water cleaning to mitigate over-nourishment and accumulation of undecomposed fish effluents, no $\mathrm{pH}$ buffering solution was added to it. There is no environmental temperature control letting the crops and aquaponic water be affected by varying ambient temperature.

Plant phenotypic images were captured thrice a week (Monday, Wednesday, and Saturday) for 6 weeks using IP Logitech camera 1080p. The generated image is configured to have $3000 \times 3000$ pixels (1:1 aspect ratio) spatial resolution. Red-colored textured clothe was used as the background (nonvegetative) of the lettuce canopy (vegetative) to increase the contrast of color distinction. Overall, there is a total of 720 lettuce phenotypic images used in this study (Table 1).

Table 1 Summary of lettuce image collection under red-blue and white light treatments with thrice a Week Image Capturing Frequency.

\begin{tabular}{cccc}
\hline Light treatment & Lettuce population & Cultivation weeks & Total images \\
\hline Red and blue spectrum & 20 & 6 & 360 \\
White spectrum & 20 & 6 & 360 \\
\hline
\end{tabular}

\section{Aquaphotomics-based water NPK detection modeling}

Aquaponic pond water samples amounting to $1.5 \mathrm{~L}$ per pond location were collected from an outdoor aquaponic pond. The elevated pond is man-made with dimensions of $10 \times 5 \times 5 \mathrm{~m}^{3}$ and the wall material is concrete. To not greatly affect the nutrient concentrations in water using fish pellets, Azolla pinnata was fed to the fishes. Two zones namely, middle (zone 1) and sidewalls (zone 2), were considered in water extraction. Zone 1 was stratified into 3 layers: Water surface (location A), middle layer (location B), and bottom layer (location C). Zone 2 was stratified into 2 layers: The waterater surface (location D) and the middle layer (location E). The basis of this water layer zoning and stratification is the sloped floor of the pond. A total of $7.5 \mathrm{~L}$ of water samples were brought back to the laboratory for aquaphotomics testing. Temperature perturbation was done using a hot plate in the temperature range of 16 to $36{ }^{\circ} \mathrm{C}$ with increments of $2{ }^{\circ} \mathrm{C}$ per water sample test resulting in 11 temperature levels. For each temperature level, 3 different water samples per extraction location were tested using UV-Vis 1900 spectrophotometer (Shimadzu) in the range of 100 to $1000 \mathrm{~nm}$. This wide spectral range is considered because no known macronutrient ion or elements is especially characterized on the selected pond water samples. At the same time, when water samples are heated in a beaker placed at the top of the hot plate, temperature, $\mathrm{pH}$, and electrical conductivity (EC) sensors connected to the Arduino UNO microcontroller were properly submerged in water to verify the changes in the water physical signatures (Figure 2). Overall, the process of temperature perturbation and transferring of water 
http://wjst.wu.ac.th

samples to $1 \mathrm{~cm}$ quartz crystal cuvette was performed 55 times. Water absorbance spectral pattern (WASP) in the form of aquagrams was generated by employing singular value decomposition in selecting the most significant pond water spectral band. After determining the spectral bands suitable for nitrate, phosphate, and potassium absorbances through wavelength sweeping aquaphotomics approach, direct spectroscopy was performed using cadmium reduction with NitraVer 5 nitrate reagent powders, Hach method with PhosVer3 phosphate reagent powders, and tetraphenylborate method using Potassium 3 reagent powder pillows for nitrate, phosphate, and potassium concentrations, respectively. The NPK concentrations were correlated and symbolically expressed using multiple linear regression.

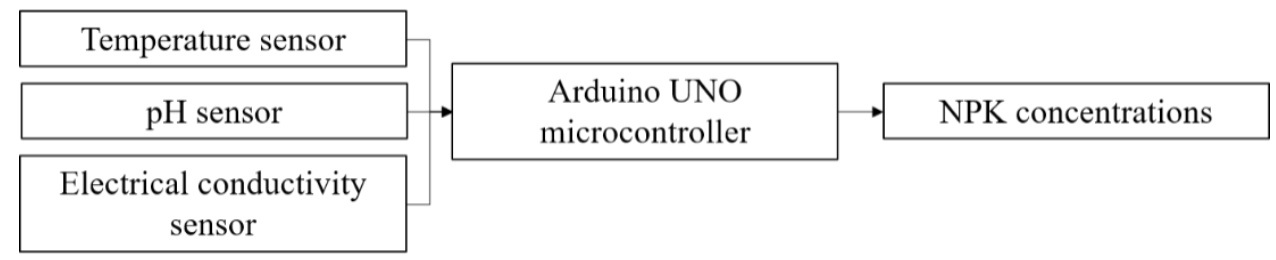

Figure 2 System instrumentation architecture in the sensor-based acquisition of physical limnological data.

\section{Vegetation Segmentation}

Vegetation segmentation is a process that subtracts the non-vegetative pixels from the image to highlight the region of interest, which is lettuce leaf canopy in this study, for further analysis. Graph-cut segmentation is performed using lazysnapping (Figure 3a) in Matlab R2020a. It starts with loading the raw digital image, transforming the RGB image into CIELab (Figure 3b), foreground and background lazysnapping with superpixels of 45,100, image masking (Figure 3c), image filtering using hole fillings, and removal of unconnected regions with less than 150 pixels and overlaying of the masked image and annotation (Figure 3d). The range for $\mathrm{L}, \mathrm{a}^{*}$, and $\mathrm{b}^{*}$ components is 0 to $100,-86.1827$ to 98.2343 , and -107.8602 to 94.4780 , respectively. The annotated image is the ground truth image that will be the basis for comparing the results of color space analysis for verification.

Each raw image has $27 \times 10^{6}$ pixels. The binary image result of each color space was evaluated using sensitivity and specificity. In the context of color space analysis in computer vision, sensitivity describes how well the color space identifies a vegetation pixel as part of the vegetative object. It is mathematically defined by the ratio of true positive (TP) and the sum of TP and false-negative (FN) (1). Specificity determines how well the specific color space identifies a non-vegetative pixel and is mathematically defined as the ratio of true negative (TB) and the sum of TN and false positive (FP) (2). Four color spaces were discriminated namely, RGB (Figure 3e), HSV (Figure 3f), CIELab (Figure 3g), and $\mathrm{YCbCr}$ (Figure 3h). The channel thresholds configured for the RGB color space are 64 to 255 for red, 125 to 255 for green, and 0 to 139 for blue. The channel thresholds configured for the HSV color space are 0.098 to 0.514 for hue and 0 to 1 for both saturation and value. The channel thresholds configured for the CIELab color space are 1 to 100 for lightness, -23 to 4.436 for red to green chromaticity, and 2.979 to 59.418 for blue to yellow chromaticity. The channel thresholds configured for the $\mathrm{YCbCr}$ are 46 to 232 for the luma component, 0 to 94 for the blue-difference component, and 0 to 255 for the red-difference component. These thresholds correspond to the lettuce leaf canopy. The color space with the highest sensitivity and specificity was selected for vegetation segmentation in batch processing. This consideration is necessary because the selected lettuce crops in this study were grown in white, red, and blue lights separately.

$$
\begin{aligned}
& \text { Sensitivity }=\mathrm{TP} / \mathrm{TP}+\mathrm{FN} \\
& \text { Specificity }=\mathrm{TN} / \mathrm{FP}+\mathrm{TN}
\end{aligned}
$$


http://wjst.wu.ac.th

\section{Spectro-textural-morphological leaf phenotype signature extraction and selection}

The architecture of the leaf canopy was characterized by extracting its spectral, texture, and morphology signatures. The RGB, HSV, L*a*b*, and $\mathrm{YCbCr}$ reflectances were quantified per image using the masked color space image, then, getting the mean value to generate the numerical value. Gray level co-occurrence matrix (GLCM) features namely, contrast, correlation, energy, entropy, and homogeneity were extracted using the binarized image. The only morphological feature extracted is the leaf canopy area $\left(\mathrm{cm}^{2}\right)$ by multiplying the leaf area in pixel to a conversion factor of $6.60847 \mathrm{e}^{-6}$ derived by using a 1 inch by 1 -inch plate captured by the same image acquisition system by the lettuce leaf canopies. Overall, there are 18 leaf architectural features analyzed in this study.

Neighborhood component analysis (NCA) and ReliefF algorithm were employed for feature selection. NCA was configured using limited-memory Broyden-Fletcher-Goldfarb-Shanno (LBFGS) algorithm as the solver with hessian history size of 15 and 'weakwolfe' as the line search method. The 18feature array of leaf canopy was inputted separately to the NCA and ReliefF algorithms. Then, principal component analysis (PCA) using listwise value treatment was performed for determining the optimal minimum number of features necessary to replicate almost $100 \%$ of the original data. In this study, PCA is configured to consider components with at least 0.5 eigenvalues.
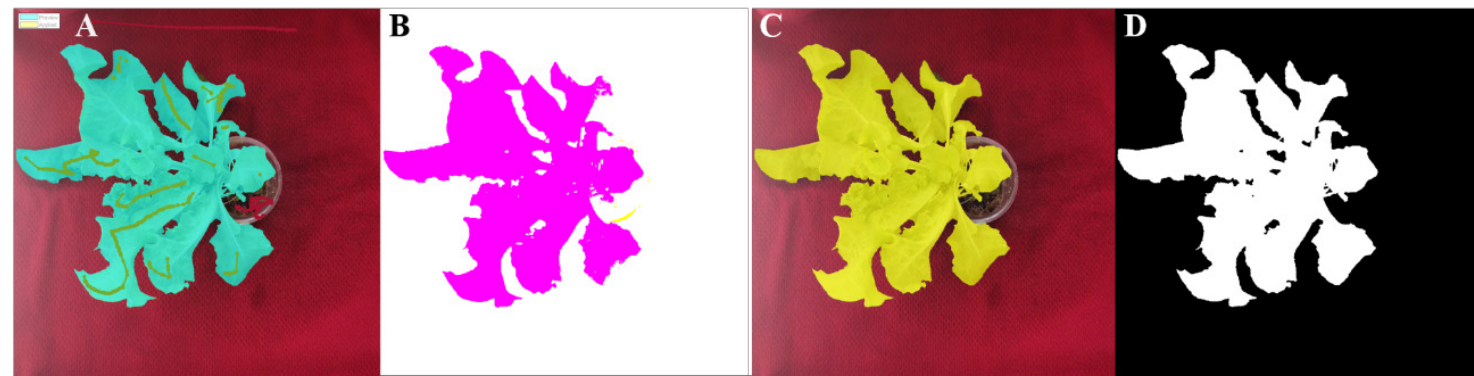

$\mathbf{E}$
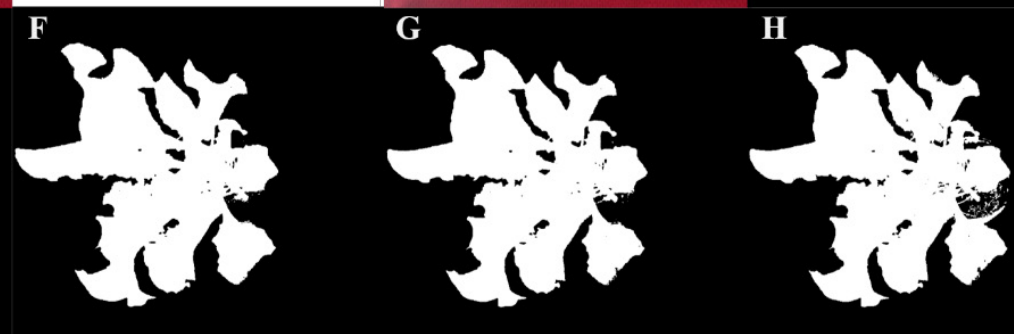

Figure 3 Graph-cut segmentation through (a) lazysnapping, (b) CIELab transformation, and (c) vegetation pixel masking that results in (d) ground truth image. Annotated color space analysis of the vegetative pixels using (e) RGB, (f) HSV, (g) CIELab, and (h) YCbCr spectrums.

\section{Aquaponic water macronutrient prediction using machine learning}

Machine learning is a subset of computational intelligence that can be constructed using featurebased or direct image-based using deep learning. In this study, feature-based machine learning modeling was performed using general processing regression (GPR), support vector machine (SVM), regressionbased decision tree (RTree), ensemble tree (TEns), and recurrent neural network (RNN) in predicting nitrogen, phosphate and potassium concentrations based on the raw 18-feature array and the hybrid PCANCA-ReliefF reduced 3-feature array. These 5 machines learning models have a different level of architecture abstraction, complexity, space consumption, and prediction performances. The GPR model using 18-features $\left(\mathrm{GRP}_{18}\right)$ as input was optimized using squared exponential as the kernel function, 
http://wjst.wu.ac.th

constant basis function with a beta of 221.4561 and sigma of 31.0754, exact predict and fit methods with an active set size of 403, and random active set method. For GPR with a 3-feature array as input $\left(\mathrm{GPR}_{3}\right)$, it was optimized using a beta of 219.8747 and sigma of 39.2028. The SVM model using 18-features as input $\left(\mathrm{SVM}_{18}\right)$ was optimized using a box constraint of 97.515 with a kernel scale of 0.90885 , epsilon of 0.78584 , bias of 116.8258 , and sequential minimal optimization (SMO) as the solver. For SVM using 3features as input $\left(\mathrm{SVM}_{3}\right)$, it was optimized using a box constraint of 5.0623 with a kernel scale of 8.012, epsilon of 0.025673 , and bias of 132.1531. The RTree model with 18-features as input (RTree ${ }_{18}$ ) was optimized using 28 minimum leaf sizes. For RTree using 3-features as input $\left(\mathrm{RTree}_{3}\right)$, it was optimized using a minimum leaf size of 48 . The TEns model with 18 -features as input $\left(\right.$ TEns $\left._{18}\right)$ was optimized using 32 minimum leaf size and least-squares boosting (LSBoost) method. For the TEns model using 3-features as input $\left(\mathrm{TEns}_{3}\right)$, it was optimized using the LSBoost method with 140 learning cycles, a learning rate of 0.10297 , and a minimum leaf size of 105 . On the other hand, the RNN models, $\mathrm{RNN}_{18}$ and $\mathrm{RNN} \mathrm{N}_{3}$ have different model configurations in predicting macronutrient concentrations as optimized by using the gravitational search algorithm (GSA).

GSA is a bioinspired algorithm (BIA) following the Newtonian laws of gravitation (3) and motion (4) [24-26]. It considers that the gravitational force (F) is directly proportional to the interaction of active mass $\left(\mathrm{m}_{\mathrm{a}}\right)$ and passive mass $\left(\mathrm{m}_{\mathrm{p}}\right)$ as affected by gravitational constant $\mathrm{G}(\mathrm{t})$ and the inverse square of the distance between the 2 mass agents. Likewise, agent acceleration (a) is affected by the interacting force of the mass of the object. In GSA, gravitational force allows each mass agent to have non-local interaction with other masses, thus, an information transfer is exhibited between 2 different masses in the isolated space system. Using Matlab R2020a platform, GSA starts with constraints and initializing object properties namely, position, inertial, active gravitational, and passive gravitational masses, randomly (Figure 4a). It should be taken into consideration that GSA considers the performance of each agent by its mass in which the lighter agent moves toward the heavier agent as affected by the gravitational force. This GSA optimization technique is used to minimize the fitness function for NPK concentrations (5-7). The developed fitness functions have the number of hidden artificial neurons on the $1^{\text {st }}, 2^{\text {nd }}$ and $3^{\text {rd }}$ layers $\left(\mathrm{N}_{1}, \mathrm{~N}_{2}, \mathrm{~N}_{3}\right)$ of the RNN model. For this experiment, $\mathrm{N}_{\mathrm{s}}$ has been configured to have a value in the range of 25 to 1000. The mean square error (MSE) exhibited by a dummy RNN model is intended to be approaching 0 with a correspondingly low number of hidden artificial neurons. Using the NPK concentration fitness functions, the gravitational and inertial masses were evaluated as they also control the agent velocity and acceleration in specific search space dimensions. Then, the position of the agents is continuously updated by basing on the heavier agent mass and it converges when the stopping criteria of 250 iterations were reached. The recorded global best fitness is the local best fitness in the last iteration during convergence, hence, its position is considered as the optimum global solution for the provided NPK concentration fitness functions. Hence, GSA optimized both $\mathrm{RNN}_{18}$ and $\mathrm{RNN}_{3}$ to have 487-356-67 hidden artificial neurons $\left(\mathrm{N}_{1}-\mathrm{N}_{2}-\mathrm{N}_{3}\right)$ for the nitrate concentration prediction, 465-422-12 neuron architecture for phosphate concentration prediction, and 963-110-32 neuron architecture for potassium concentration prediction (Figure 5).

$$
\begin{aligned}
& \mathrm{F}=\mathrm{G}(\mathrm{t})\left(\mathrm{m}_{\mathrm{a}} \mathrm{m}_{\mathrm{p}} / \mathrm{r}^{2}\right) \\
& \mathrm{a}=\mathrm{F} / \mathrm{m} \\
& \mathrm{MSE}_{\mathrm{N}}=1128.1+0.495 \mathrm{~N}_{1}-0.329 \mathrm{~N}_{2}+0.98 \mathrm{~N}_{3} \\
& \mathrm{MSE}_{\mathrm{P}}=1103.3+0.3936 \mathrm{~N}_{1}-0.184 \mathrm{~N}_{2}+0.701 \mathrm{~N}_{3} \\
& \mathrm{MSE}_{\mathrm{K}}=1299.9+0.525 \mathrm{~N}_{1}-0.406 \mathrm{~N}_{2}+1.16 \mathrm{~N}_{3}
\end{aligned}
$$




\section{A}

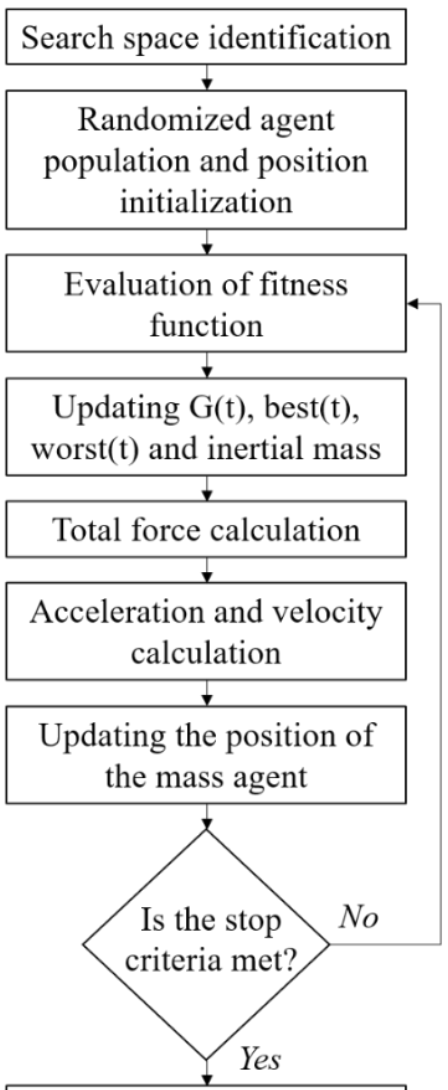

Optimum solution

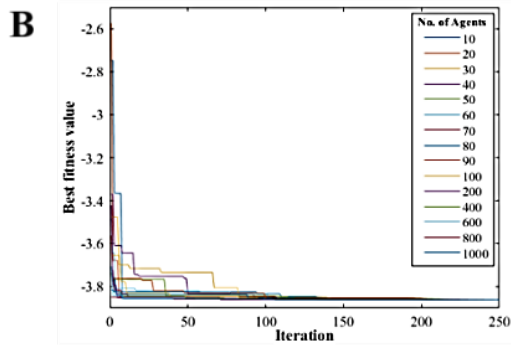

C

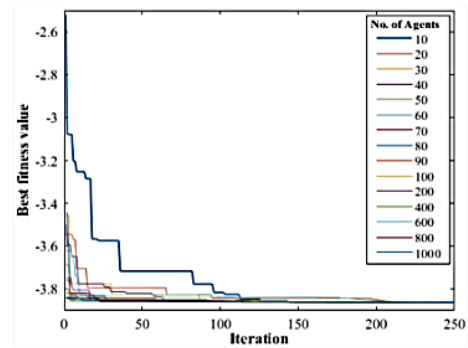

D

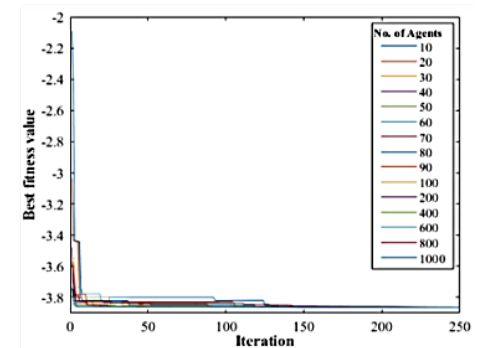

Figure 4 Gravitational search algorithm for optimizing the mean square error of the NPK concentration fitness models (a). The best fitness curve for (b) nitrate, (c) phosphate, and (d) potassium concentrations as optimized by changing the number of agents from 10 to 1000 .

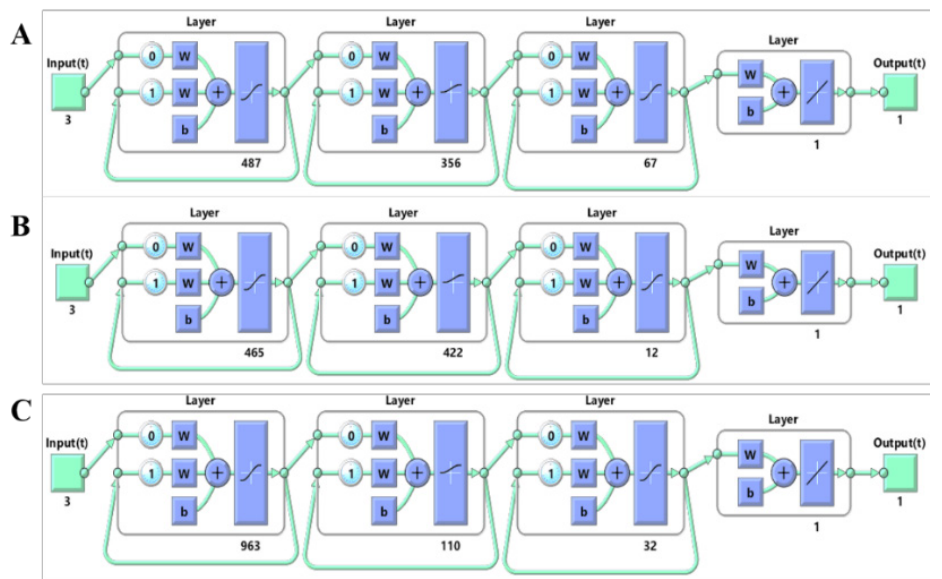

Figure 5 Recurrent neural network architecture with GSA-optimized number of hidden artificial neurons for (a) nitrate, (b) phosphate, and (c) potassium concentration predictions. 


\section{Model evaluation metrics and statistical analysis}

The performance of the developed feature-based machine learning models was evaluated using RMSE, $R^{2}, M A E$, and inference time from training, validation, and testing stages. Significant data correlations were considered at $\alpha \leq 0.05$ using Minitab 19 (Minitab, LLC).

\section{Results and discussion}

Color space analysis

The resulting color marker mean values serving as the threshold for the lettuce pixel region in RGB, HSV, CIELab, and YCbCr color spaces are presented in Table 2. Through color space analysis, the most suitable color space for vegetation segmentation in this study was determined. All pre-selected color spaces have very high specificity performance with CIELab as the most accurate with $99.99 \%$ (Table 3). It means that any of the color spaces can be considered to correctly selecting non-vegetation pixels. However, the accuracy in determining the ROI vegetation pixels must be strictly considered. In this case, CIELab extends its quality performance to $98.721 \%$ sensitivity which is $41.754 \%$ higher than $\mathrm{YCbCr}$ (Table 3). It was observed that $\mathrm{RGB}$ and $\mathrm{YCbCr}$ color spaces are sensitive to white chromaticity as resembled by the plastic cups with incident light (Figure 3). Deconstructed binarized images were rendered and a high amount of non-vegetation pixels were included in the annotated image. The representative annotated images of HSV and CIELab color spaces (Figures $\mathbf{3 f}$ and $\mathbf{3 g}$ ) were very close to the ground truth image resulting in a $4.361 \%$ difference for sensitivity. Overall, CIELab is the optimal color space for vegetation segmentation in this application as it exhibited distinct clustering in the regions of the lettuce leaf, and red mantle, rockwool, and plastic cups. Moreover, CIELab is preferable to the ground truth approach of graph-cut segmentation because the process of doing it is a lot faster without the need to do the manual lazysnapping step and the system space-consuming super pixels. In contrast, $\mathrm{YCbCr}$ is the chosen color space when the image background is brown resembling the natural soil color [27].

Table 2 Color marker mean values for each color space in the vegetation and non-vegetation regions.

\begin{tabular}{cccc}
\hline Color space & Color component & Lettuce region & Non-vegetation region \\
\hline RGB & R & 159.5 & 32 \\
& G & 190 & 62.5 \\
$\mathrm{BSV}$ & $\mathrm{B}$ & 69.5 & 194.5 \\
& $\mathrm{H}$ & - & - \\
$\mathrm{S}$ & 0.5 & 0 \\
$\mathrm{CIELab}$ & $\mathrm{S}$ & 0.5 & 0 \\
& $\mathrm{~V}$ & 50.549 & 0.549 \\
& $\mathrm{~L}$ & -9.7355 & 52.213 \\
$\mathrm{YCbCr}$ & $\mathrm{a}^{*}$ & 3.7025 & -48.5105 \\
& $\mathrm{~b}^{*}$ & 139 & 23 \\
& $\mathrm{Y}$ & 47 & 172 \\
\hline
\end{tabular}

Table 3 Average sensitivity and specificity of various color spaces.

\begin{tabular}{ccc}
\hline Color space & Sensitivity (\%) & Specificity (\%) \\
\hline RGB & 76.332 & 99.781 \\
HSV & 94.360 & 99.954 \\
CIELab & 98.721 & 99.990 \\
YCbCr & 56.967 & 99.663 \\
\hline
\end{tabular}




\section{Significant lettuce leaf signatures}

The 3 clusters of leaf signatures extracted in this study are the spectral, texture, and morphological features. Each component was extracted from the CIELab-thresholded masked image highlighting the lettuce vegetative pixel regions. For nitrate concentration, among the 18 extracted leaf features, $a^{*}$, blue, and $\mathrm{Cr}$ are the most significant components that can easily contribute to correct nutrient concentration prediction (Figure 6a). For phosphate concentration prediction, $\mathrm{a}^{*}$, blue, and $\mathrm{Cb}$ components weighted the most impactful as shown in the weight spectrum (Figure 6b). For potassium concentration prediction, blue, a*, and Cr garnered the highest weights (Figure 6c). The PCA-projected eigenvalue of the 18 leaf canopy features confirmed that 3 components exhibited $98.9286 \%$ of the total variability of the original dataset. This entails that a reduction from 18 to 3 features is worth it and a good approach to improve the computational cost of the model. Across the 3 weight spectrums, it appears that red, blue, saturation, $a^{*}$, $\mathrm{Cb}, \mathrm{Cr}$, and energy has considerable spread and spike that indicates its relevance with the nutrient concentration being predicted. It is noticeable that most of the highly relevant components are spectral signatures of the leaf. This profoundly signifies that macronutrients have a direct effect to crop leaf color for both white and red-blue light treatments. It is an agreement with [28] that macronutrient deficiency, especially in potassium, easily results in discoloration or initiation of leaf spots. Phosphate deficiency results in dark green color on the upper portion of the leaves and bronze to purple on the lower surfaces. Likewise, lack of nitrogen in the water system for aquaponics induced the crop leaves to exhibit chlorosis or the yellowing of its leaf tissue as it is part of the chlorophyll molecule that is responsible for the green color of the plants. To standardize the input features for 3-feature array machine learning models, blue, $\mathrm{a}^{*}$, and $\mathrm{Cr}$ color components were selected based on the recommendation of the hybrid PCA-NCAReliefF technique. In this study, the blue, green-red component and red minus luma color features of lettuce leaf canopy deliver a high sensitivity prediction performance for nutrient concentrations in water solution.

The blue component of lettuce cultivated in white and red-blue treatment has a weak-to-weak strong positive correlation with water nitrate and potassium concentrations and weak negative correlation to phosphate concentration (Table 4). The green-red component of lettuce has a weak negative correlation with nitrate and potassium concentrations, and a weak positive correlation with phosphate (Table 4). The blue component from the RGB color space has an inverse relationship with the green-red component of the CIELab color space. The red minus luma component of $\mathrm{YCbCr}$ color space has a weak negative correlation with phosphate and a weak positive correlation with potassium concentrations for both light treatments. Interestingly, $\mathrm{Cr}$ has different characterization for nitrate concentration in the red blue (weak negative) and white (weak positive) light treatments. Among the 3 macronutrients, fluctuations of potassium concentration in the water source for the hydroponic grow bed of lettuce has the most substantial impact on leaf spectral information.

Table 4 Correlation coefficient from Pearson's correlation analysis of water NPK, and blue, a* and Cr lettuce leaf spectral signatures.

\begin{tabular}{cccccc}
\hline Light treatment & $\mathbf{n}$ & Macronutrient & $\mathbf{B}$ & $\mathbf{a}^{*}$ & $\mathbf{C r}$ \\
\hline Red and blue spectrum & 360 & $\mathrm{~N}$ & 0.315 & -0.270 & -0.340 \\
& & $\mathrm{P}$ & -0.245 & 0.183 & -0.231 \\
White spectrum & $\mathrm{K}$ & 0.123 & -0.231 & 0.175 \\
& \multirow{3}{*}{360} & $\mathrm{~N}$ & 0.444 & -0.276 & 0.192 \\
& & $\mathrm{P}$ & -0.337 & 0.224 & -0.114 \\
& & $\mathrm{~K}$ & 0.492 & -0.271 & 0.284 \\
\hline
\end{tabular}


A

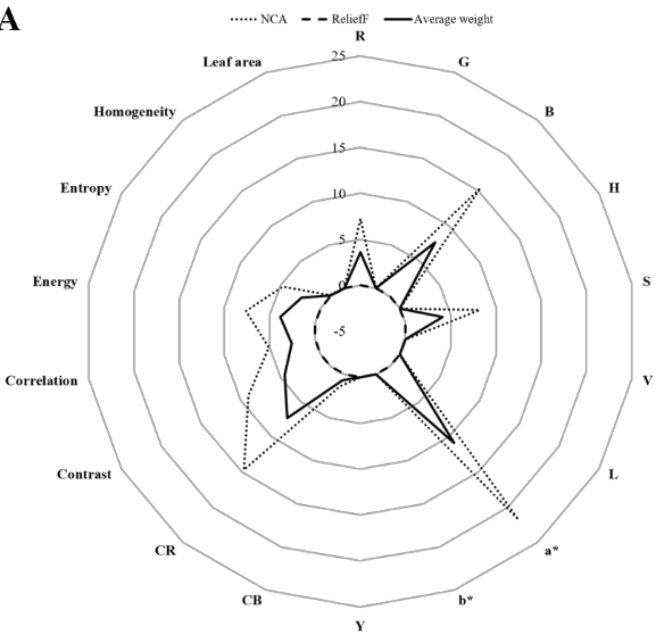

C

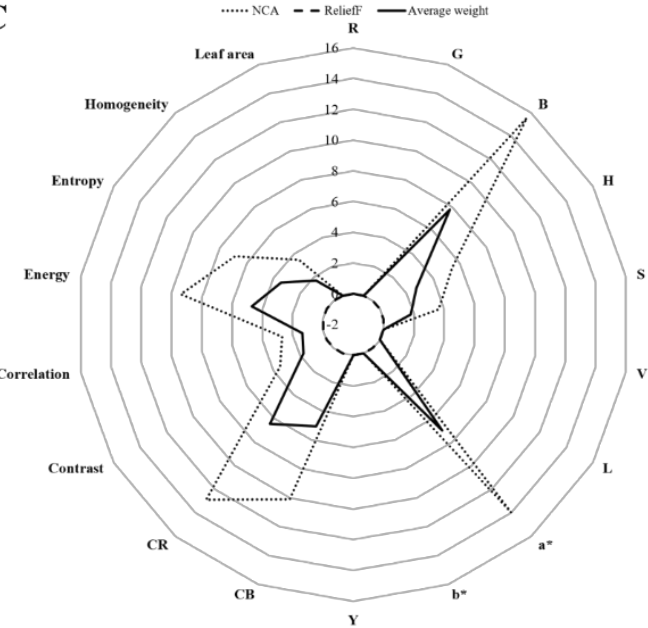

B

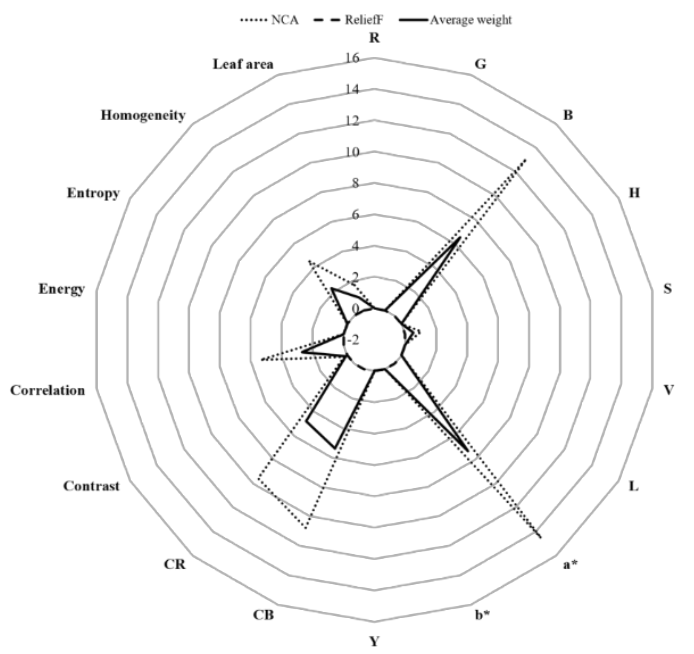

D

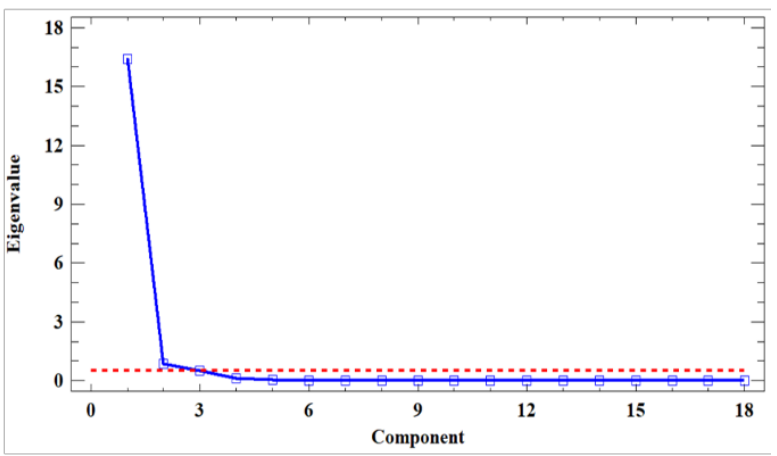

Figure 6 Weight spectrum generated using hybrid NCA and ReliefF for (a) nitrogen, (b) phosphate, (c) potassium spectro-textural-morphological feature selection, and (d) eigenvalue projection of each predictor using PCA.

\section{Extracted macronutrient biomarkers and GSA-based optimization of RNN model}

The constructed aquagram defining the extracted macronutrient biomarkers from aquaponic pond water solution indicates that nitrate, phosphate, and potassium concentration can be extracted through aquaphotomics means in the ultraviolet, visible light, and near-infrared spectrums (Figure 7a). The 11 water absorbance wavelengths are 195, 200, 205, 210, 215, 415, 235, 275, 415, 765, 835, and $840 \mathrm{~nm}$. These wavelengths are the characteristic spectral coordinates that resemble the water spectral absorbance pattern. Since the pond water has been nourished by fishes for more than 3 months before the start of lettuce cultivation, water is rich with essential nutrients for plant intake. Water samples from 5 different pond locations in the aquaponic pond generated a water spectral absorbance pattern that highlights the 205,840 , and $765 \mathrm{~nm}$ wavelengths across the 100 to $1000 \mathrm{~nm}$ span that are considered as the wavelength protocol for nitrate, phosphate, and potassium concentrations respectively [29-31]. Different organic compounds have been detected also in the aquagram (Figure 7a) but only NPK are important in this study. Nitrate, phosphate, and potassium concentrations are highly present in the water surface in the 
http://wjst.wu.ac.th

middle section (location A) of the pond. The bottom layer (location C) of the pond is rich in phosphate and potassium. The pond sidewalls (locations D and E) happened to have a high amount of nitrate ions. This slightly uneven distribution of macronutrients is exhibited in the artificial aquaponic pond because of the presence of large stones at the pond floor and poor aeration. However, in this study, this setup is acceptable as different macronutrient concentrations must be extracted from water samples.

The impact of increasing artificial hidden neurons on the 3 hidden layers of the RNN model through gravitational search algorithm was visualized in separate 4 dimensions for nitrate, phosphate, and potassium concentration prediction models (Figures 7(b) - 7(d)). The fitness function for nitrogen (5), phosphate (6), and potassium (7) concentration MSE prediction characterization as affected by the combination of the 3 hidden layers of artificial hidden neurons were inferred for a minimization optimization. This means that the position of the global minimum MSE attainable in the entire search space is considered as the solution to the problem or the optimum value of the artificial neurons on the 3 hidden layers. The implementation of the gravitational search algorithm in (1) resulted in Figure $\mathbf{7 b}$ that projects the combination of 487-356-67 $\left(\mathrm{N}_{1}, \mathrm{~N}_{2}, \mathrm{~N}_{3}\right)$ to have the global minimum MSE, thus, considered as the ideal network for nitrate prediction. Concerning the number of agents that is the only hyperparameter that was tweaked in the optimization process, the fitness function value substantially approaches 0 as the number of agents is configured smaller. Hence, for the RNN nitrate concentration model, the ideal number of agents is 60 . For the phosphate prediction RNN model, 10 agents as the starting masses yielded the global minimum of -3.19389 MSE with optimum artificial neuron combination of 465-422-12 (Figure 7c). For the potassium prediction RNN model, 50 agents resulted in the global minimum of -3.1259 MSE with an optimum artificial neuron combination of 963-110-32 (Figure 7d). With other neuron combinations, it is evident that the trend is erratic and there is no specific scheme for minimizing the MSE value of the fitness functions (5), (6), and (7) other than by using the intelligent gravitational search optimization algorithm. Hence, using GSA the number of hidden artificial neurons was successfully optimized for all RNN models. 
A

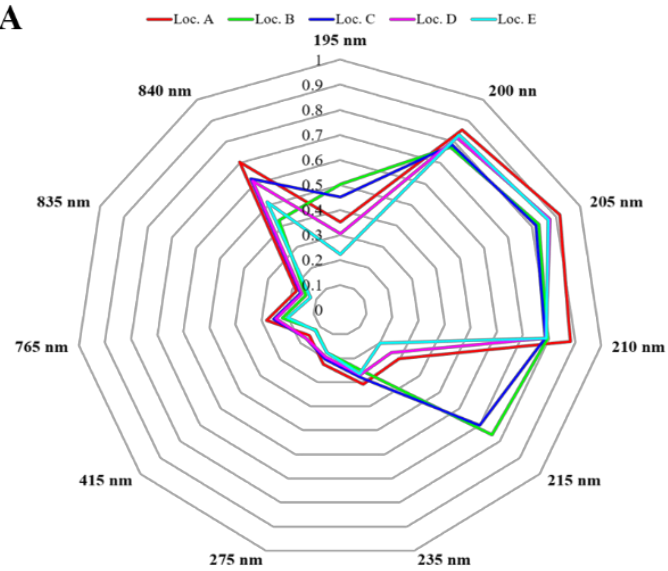

C

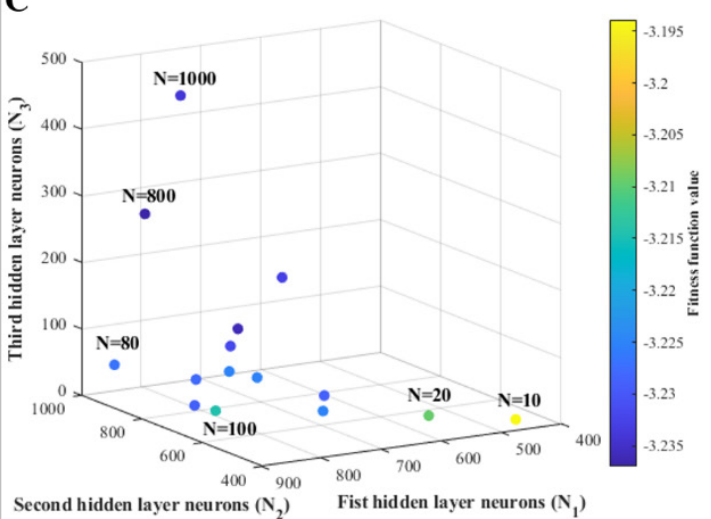

B

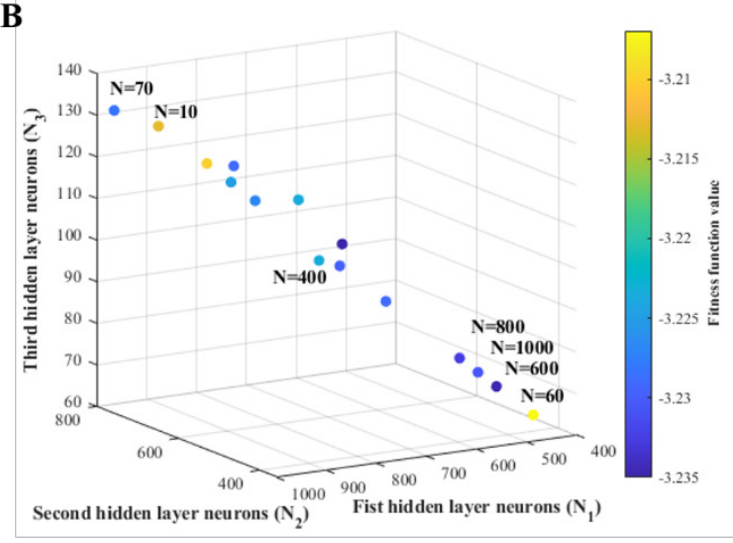

D

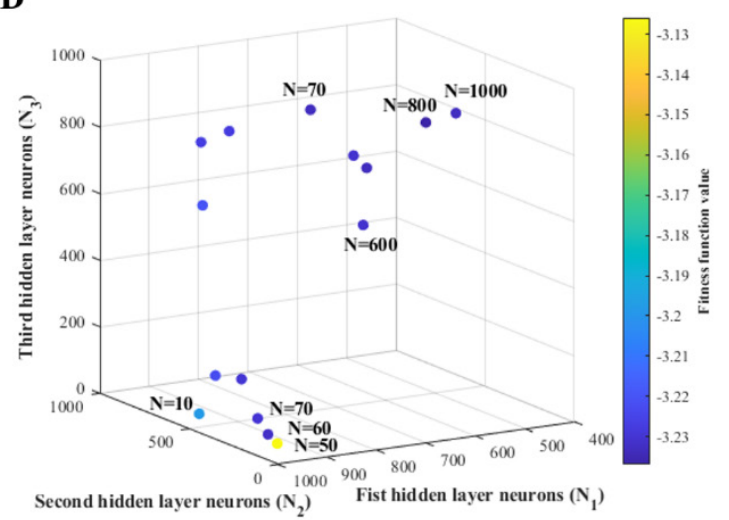

Figure 7 (a) Aquagram of significant water spectral absorbance pattern in the UV-V is spectrum using aquaphotomics and the impact of increasing artificial hidden neurons on the $1^{\text {st }}, 2^{\text {nd }}$, and $3^{\text {rd }}$ hidden layers of the recurrent neural network to the fitness function value in predicting (b) nitrate, (c) phosphate and (d) potassium concentrations.

\section{Water macronutrients concentration prediction}

The use of feature-based machine learning models namely generalized processing regression, regression-based support vector machine, regression-based decision tree, ensemble tree, and recurrent neural network, was effectively employed in this study to predict the macronutrient concentration present in water system based on the leaf signatures of lettuces that are cultivated using aquaponic approach (Figure 8). For nitrate concentration prediction, the hybrid NCA-ReliefF-RNN model $\left(\mathrm{RNN}_{3}\right)$ bested out other AI networks with an accuracy of $93.61 \%$ and an inference time of $7 \mathrm{~s}$ (Table 5). Even without feature selection to reduce the original 18 lettuce leaf signatures, $\mathrm{RNN}_{18}$, still exhibited high accuracy of $83.49 \%$ in the testing phase. $\mathrm{GPR}_{18}, \mathrm{RTree}_{18}, \mathrm{RTree}_{3}, \mathrm{TEns}_{18}$, and $\mathrm{TEns}_{3}$ have acceptable accuracy performances from training to validation stages, but all of these models failed to have good sensitivity for a unique dataset in the testing phase. $\mathrm{GPR}_{18}$ even resulted in $1.79 \%$ accuracy during testing. On the other hand, both RNN variants increased their accuracy from training to validation by a factor of 1.7491 and 2.0908 , respectively. $\mathrm{RNN}_{3}$ somehow managed to improve it in the testing phase by a factor of 1.0296 . $\mathrm{RNN}_{3}$ is $53.80 \%$ faster than $\mathrm{SVM}_{3}$ as the slowest model for nitrate concentration prediction. For phosphate concentration prediction based on lettuce leaf signatures, $\mathrm{RNN}_{18}$ exhibited the best performance of $84.03 \%$ accuracy (Table 5). RTree and TEns variants have very high accuracy during the 
training stage but suddenly dropped down by a factor of 2.6958 in the validation and 1.0774 in the testing stage. On the other hand, RNN variants have increased their accuracy in predicting phosphate concentration by a factor of 3.1391. In the testing stage, $\mathrm{RNN}_{18}$ decreased by 1.0966 and $\mathrm{RNN}_{3}$ resulted in a better increase performance of 1.0087 that is $84.03 \%$. The inference time of $\mathrm{RNN}_{18}$ is surprisingly faster than $\mathrm{RNN}_{3}$ despite that the latter has 3 input features only. The possible reason for this unconventional inference time based on the complexity of the input features is the internal architecture of the RNN variants as they were both optimized by GSA and resulted in the same network model. Hence, $\mathrm{RNN}_{3}$ is the preferred model because of its higher accuracy. For the prediction of potassium concentration in water source based on lettuce leaf signatures, $\mathrm{RNN}_{3}$ bested out other models with an accuracy of $91.39 \%$ (Table 5). SVM variants have consistently worst predictions from training to the testing stage. RNN3 has increased its accuracy performance by a factor of 3.7079 from training to testing. Among the 3 GSA-RNN 3 macronutrient prediction models, the nitrate model estimates the fastest and the potassium model estimates the slowest. The target value versus GSA-RNN 3 predictions for nitrate, phosphate, and potassium estimations separately signifying that the sensitivity of the developed models is acceptable for smart farm applications (Figures 8(a) - 8(c)). By comparing the ground truth with the obtained predictions from the GSA-RNN $\mathrm{R}_{3}$ model, it is possible to notice that the developed model has high efficiency in estimating water macronutrient concentrations by just using a consumer-grade camera and looking on to leaf photosynthetic signatures. These facts reinforced the applicability of the GSA$\mathrm{RNN}_{3}$ machine learning model with a high degree of accuracy, sensitivity, and efficiency for low computational cost systems. Hence, the NCA-ReliefF-GSA-RNN model formally encapsulates the wNPK model.

After several explorations in using gravitational search for optimizing feature-based recurrent neural network in predicting macronutrient concentration in water, this study successfully developed a hybrid NCA-ReliefF-GSA-RNN that can estimate nitrate, phosphate, and potassium concentrations with the accuracy of $93.61,84.03$, and $91.39 \%$ respectively. There are previously published studies about water nutrient concentration estimation (Table 6). The trend of techniques in determining water nutrient concentration seems to be moving from plain laborious laboratory approaches [13], the aid of electronic sensors [11] and hybrid machine learning models based on sensor acquired data [14,18,19,23]. The study done by Concepcion II et al. (2020) employed the integration of aquaphotomics with multigene symbolic regression genetic programming (MSRGP) to predict total organic carbon (TOC) and hydrogen from the same aquaponic pond water samples used in the present study [18]. The basis for these predictions is temperature and electrical conductivity sensor data. The same pond water extraction location is evident in the phosphate concentration prediction based on temperature, EC, and $\mathrm{pH}$ sensor readings integrated with an adaptive neuro-fuzzy inference system (ANFIS) [19]. The resulting accuracy of ANFIS is $1.19 \%$ behind the result of the NCA-ReliefF-GSA-RNN model. The closest accuracy to the developed models was done by Patokar and Gohokar (2018) that used a color sensor deployed in clean white containers and light intensifiers to see the difference of color distribution in the water when the computed amount of NPK chemicals were diluted into it [23]. However, its major drawback is that in the actual scenario, NPK fertilizers are not encouraged in an aquaponic cultivation setup such as presented in the current study. In ideal aquaponics, macronutrients are solely based on the interaction of microorganisms present in the water system and the fish effluents. The developed hybrid NCA-ReliefF-GSA-RNN is much reliable in the industrial-wide application as crop leaves are a direct indicator of the number of micronutrients in the water system. Although the NCA-ReliefF-GSA-RNN model for nitrate and potassium have a fraction lower than [23], still the prior has the advantage as it has been deployed in the actual smart farm setup. Likewise, the NCA-ReliefF-GSA-RNN requires only a digital RGB camera for the acquisition of images while [23] entails one color sensor for each nutrient to be estimated. The technique developed concerning the NCA-ReliefF-GSA-RNN model works differently as it focuses on crop leaf canopy while all mentioned studies in Table 6 have direct work out with the water samples. Efficiency and economical wise, the wNPK (NCA-ReliefF-GSA-RNN) model has the advantage. 
A

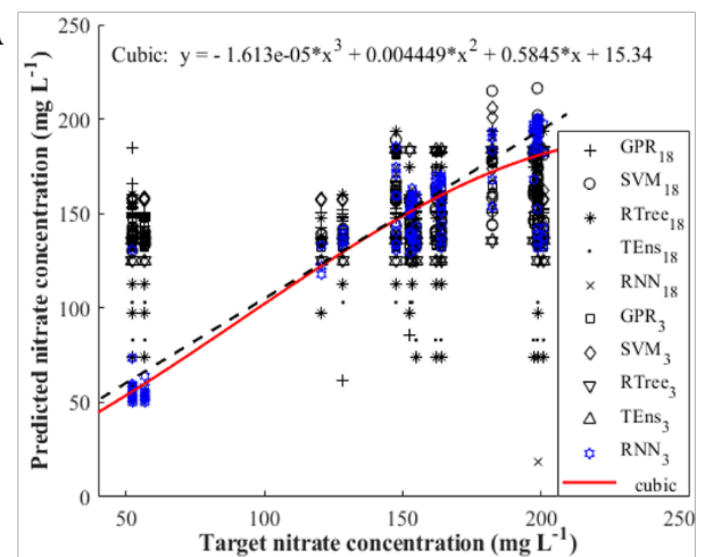

B

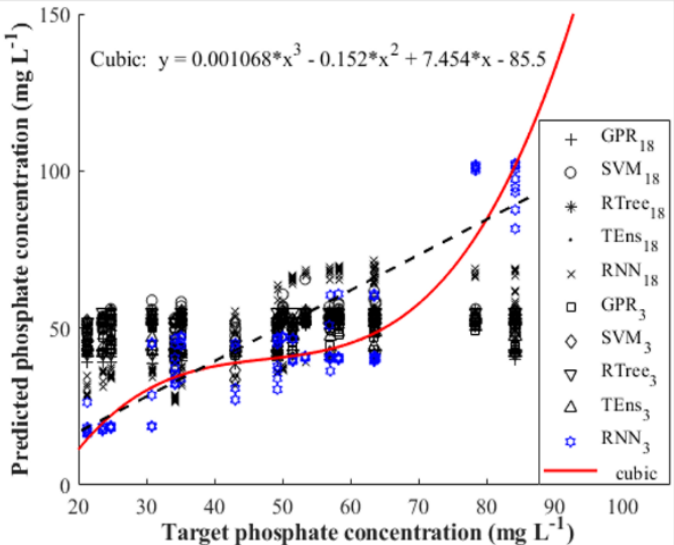

C $\overparen{\sim} 280$ - Cubic: $y=+5.945 e-05^{*} x^{3}+0.03706^{*} x^{2}-6.578 * x+496.6$

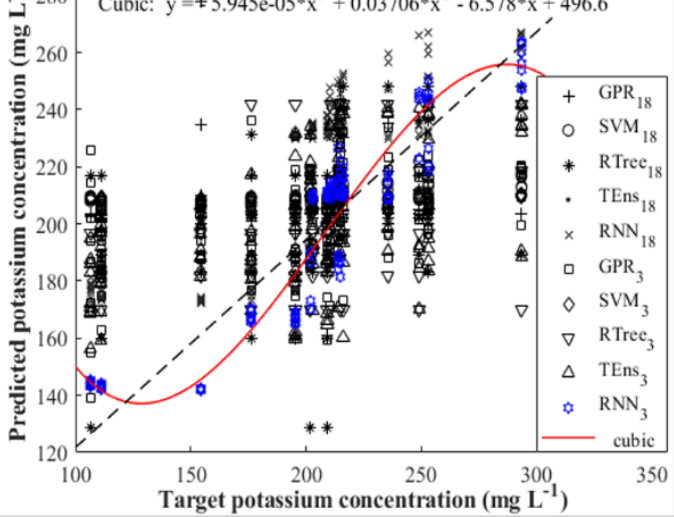

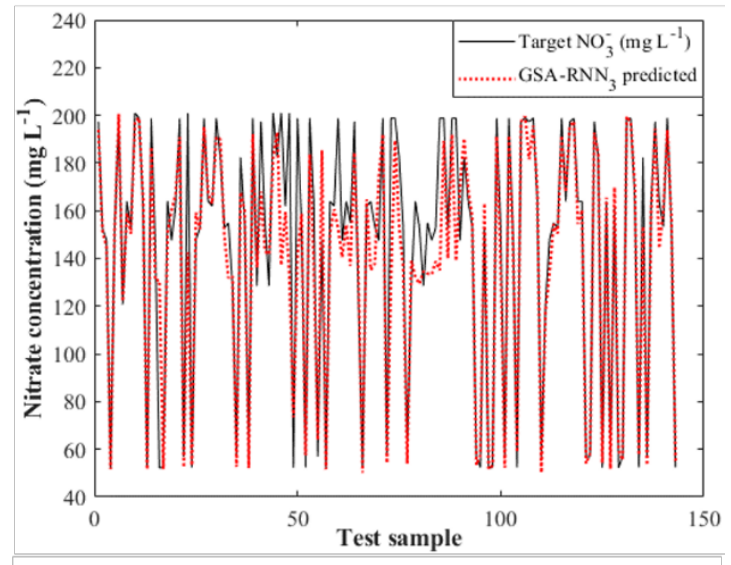
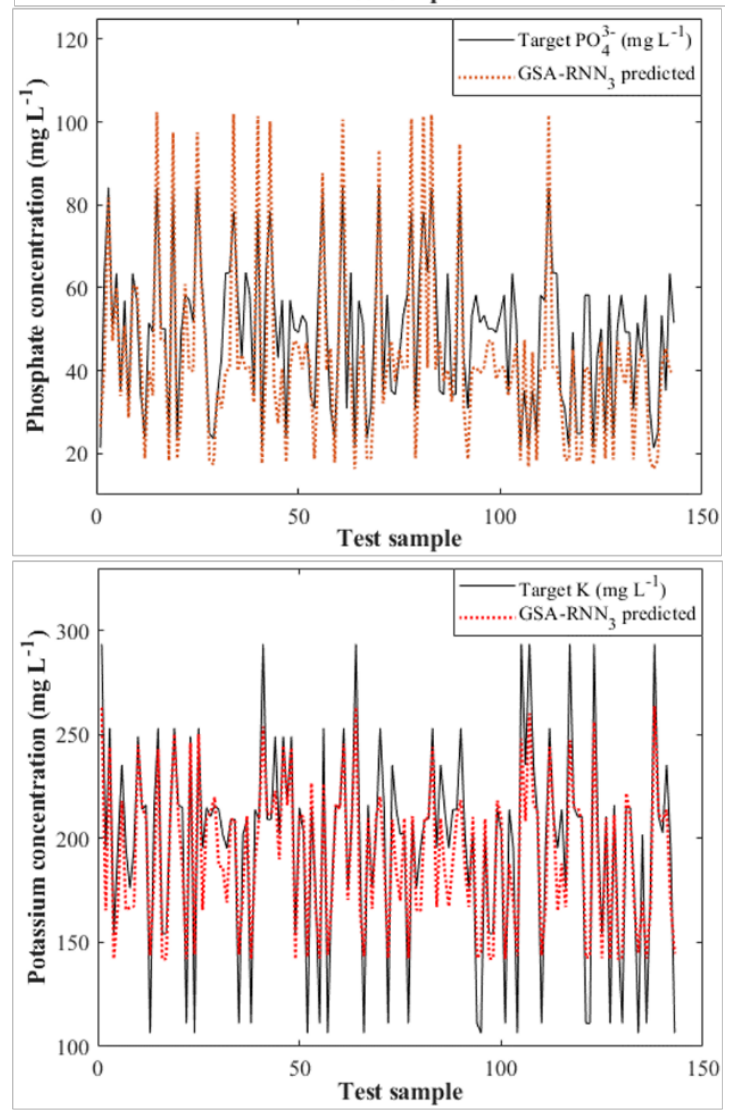

Figure 8 Prediction curve of the developed models as discriminated with the target value of (a) nitrate, (b) phosphate, and (c) potassium concentrations in the water system. 
http://wjst.wu.ac.th

Table 5 Model performance evaluation in predicting nitrogen, phosphate, and potassium concentrations in water system using feature-based machine learning models based on lettuce leaf signatures.

\begin{tabular}{|c|c|c|c|c|c|c|c|c|c|c|c|}
\hline \multirow{2}{*}{ Model } & \multirow{2}{*}{$\begin{array}{c}\text { No. of } \\
\text { features }\end{array}$} & \multicolumn{3}{|c|}{ Training } & \multicolumn{3}{|c|}{ Validation } & \multicolumn{4}{|c|}{ Testing } \\
\hline & & RMSE & $\mathbf{R}^{2}$ & MAE & RMSE & $\mathbf{R}^{2}$ & MAE & RMSE & $\mathbf{R}^{2}$ & MAE & $\begin{array}{c}\text { Inference } \\
\text { time (s) }\end{array}$ \\
\hline \multicolumn{12}{|c|}{ Nitrate concentration prediction } \\
\hline GPR & 18 & 34.57 & 0.71 & 25.69 & 0.01 & 1.00 & 0.00 & 53.33 & 0.02 & 40.45 & 35.80 \\
\hline GPR & 3 & 42.49 & 0.48 & 32.62 & 41.87 & 0.47 & 32.18 & 45.95 & 0.49 & 36.06 & 46.83 \\
\hline SVM & 18 & 45.98 & 0.40 & 32.18 & 42.45 & 0.44 & 33.10 & 46.37 & 0.49 & 36.86 & 185.03 \\
\hline SVM & 3 & 47.05 & 0.38 & 32.63 & 47.14 & 0.34 & 32.26 & 52.44 & 0.33 & 36.88 & 376.61 \\
\hline RTree & 18 & 15.00 & 0.95 & 8.57 & 33.99 & 0.70 & 24.91 & 43.59 & 0.57 & 31.34 & 14.90 \\
\hline RTree & 3 & 21.59 & 0.89 & 13.30 & 39.76 & 0.54 & 31.42 & 44.47 & 0.53 & 36.36 & 25.58 \\
\hline TEns & 18 & 0.15 & 1.00 & 0.11 & 35.18 & 0.67 & 26.30 & 43.47 & 0.56 & 32.15 & 17.02 \\
\hline TEns & 3 & 1.48 & 1.00 & 1.13 & 39.76 & 0.54 & 31.42 & 44.47 & 0.53 & 36.36 & 28.54 \\
\hline $\mathrm{RNN}$ & 18 & 41.41 & 0.52 & 32.21 & 20.63 & 0.90 & 13.85 & 29.33 & 0.83 & 16.48 & 7.00 \\
\hline RNN & 3 & 43.55 & 0.43 & 33.87 & 20.07 & 0.91 & 13.05 & 19.25 & 0.94 & 11.57 & 7.00 \\
\hline \multicolumn{12}{|c|}{ Phosphate concentration prediction } \\
\hline GPR & 18 & 16.41 & 0.39 & 12.71 & 15.67 & 0.43 & 12.31 & 16.54 & 0.34 & 12.73 & 47.27 \\
\hline GPR & 3 & 16.58 & 0.36 & 12.94 & 15.98 & 0.39 & 12.72 & 16.69 & 0.32 & 12.97 & 50.09 \\
\hline SVM & 18 & 17.44 & 0.23 & 13.59 & 16.78 & 0.32 & 12.84 & 17.10 & 0.30 & 12.90 & 172.19 \\
\hline SVM & 3 & 17.51 & 0.22 & 13.67 & 16.95 & 0.23 & 13.28 & 17.33 & 0.22 & 13.50 & 246.87 \\
\hline RTree & 18 & 8.16 & 0.89 & 5.19 & 16.26 & 0.32 & 12.59 & 16.59 & 0.33 & 12.56 & 24.69 \\
\hline RTree & 3 & 9.65 & 0.84 & 6.91 & 16.18 & 0.34 & 12.59 & 16.66 & 0.32 & 12.62 & 27.83 \\
\hline TEns & 18 & 0.10 & 1.00 & 0.07 & 16.26 & 0.32 & 12.59 & 16.59 & 0.33 & 12.56 & 31.27 \\
\hline TEns & 3 & 0.71 & 1.00 & 0.56 & 15.88 & 0.39 & 12.48 & 16.70 & 0.31 & 12.89 & 204.53 \\
\hline RNN & 18 & 16.92 & 0.29 & 13.30 & 9.37 & 0.88 & 7.77 & 10.96 & 0.80 & 9.65 & 7.00 \\
\hline RNN & 3 & 17.14 & 0.25 & 13.55 & 14.36 & 0.83 & 12.26 & 13.14 & 0.84 & 11.03 & 31.00 \\
\hline \multicolumn{12}{|c|}{ Potassium concentration prediction } \\
\hline GPR & 18 & 27.45 & 0.80 & 19.41 & 0.00 & 1.00 & 0.00 & 49.77 & 0.03 & 35.63 & 62.17 \\
\hline GPR & 3 & 37.79 & 0.54 & 27.22 & 29.73 & 0.73 & 20.99 & 42.76 & 0.49 & 32.24 & 50.29 \\
\hline SVM & 18 & 43.65 & 0.29 & 28.79 & 40.78 & 0.32 & 26.47 & 48.48 & 0.41 & 33.53 & 340.37 \\
\hline SVM & 3 & 44.00 & 0.25 & 28.95 & 42.10 & 0.19 & 27.67 & 49.86 & 0.19 & 34.67 & 134.70 \\
\hline RTree & 18 & 15.71 & 0.93 & 9.38 & 29.89 & 0.70 & 22.05 & 39.96 & 0.58 & 30.39 & 33.34 \\
\hline RTree & 3 & 21.07 & 0.88 & 13.43 & 32.70 & 0.62 & 24.29 & 44.37 & 0.42 & 34.79 & 25.57 \\
\hline TEns & 18 & 0.12 & 1.00 & 0.09 & 29.97 & 0.69 & 21.95 & 40.02 & 0.58 & 30.44 & 30.89 \\
\hline TEns & 3 & 1.25 & 1.00 & 0.94 & 32.03 & 0.64 & 23.90 & 42.33 & 0.49 & 33.62 & 256.14 \\
\hline RNN & 18 & 41.66 & 0.34 & 30.12 & 21.91 & 0.93 & 17.50 & 33.86 & 0.80 & 27.12 & 7.00 \\
\hline RNN & 3 & 42.84 & 0.25 & 30.45 & 22.93 & 0.87 & 21.28 & 22.22 & 0.91 & 17.83 & 67.00 \\
\hline
\end{tabular}


Table 6 Accuracy of the developed approach in determining water nutrient concentration as compared with other techniques.

\begin{tabular}{llcc}
\hline \multicolumn{1}{c}{ Author and Date } & \multicolumn{1}{c}{ Technique/Model } & Nutrient & Accuracy \\
\hline Thirunavuakkarasu et al., 2017 [11] & Sensor-based & NPK & Not provided \\
Liu et al., 2020 [13] & Diffusion method & Nitrate & Not provided \\
& Colorimetric measurement & Phosphate & Not provided \\
& Flame emission spectroscopy & Potassium & Not provided \\
Shi et al., 2017 [14] & Generalized likelihood & Nitrate & $89.00 \%$ \\
& uncertainty estimation & Phosphate & $87.00 \%$ \\
Concepcion II et al., 2020 [18] & MSRGP based on water & TOC & $92.80 \%$ \\
& temperature and EC & Hydrogen & $96.93 \%$ \\
Lauguico et al., 2020 [19] & ANFIS based on water & Phosphate & $82.84 \%$ \\
Patokar and Gohokar et al., 2018 [23] & temperature, EC, and pH & & \\
& Color sensor using a container & Nitrogen & $95.47 \%$ \\
& with clean white material & Phosphorus & $73.99 \%$ \\
& & Potassium & $96.37 \%$ \\
& NCA-ReliefF-GSA-RNN & Nitrate & $93.61 \%$ \\
& (wNPK) based on crop leaf & Phosphate & $84.03 \%$ \\
& signatures & Potassium & $91.39 \%$ \\
\hline
\end{tabular}

\section{Plant nutrient uptake response}

The plant consumes all needed nutrients from the water in an aquaponics set up to promote growth. The weekly average value of macronutrient concentration on the tank and the outlet pipe is shown in Figure 9. The nitrate $\left(\right.$ Nitrate $\left._{\text {in }}\right)$, phosphate $\left(\right.$ Phosphate $\left._{\text {in }}\right)$, and potassium $\left(\right.$ Potassium $\left._{\text {in }}\right)$ concentrations in the tank have increased from the $1^{\text {st }}$ week of cultivation to the $2^{\text {nd }}$ week by a factor of $2.5,1.24$, and 1.65 , respectively. This increase is mainly because crops do not consume a large number of nutrients for that moment as they are in the vegetative stage. However, potassium is observed to diminished from 123.9672 $\mathrm{mg} \mathrm{L}^{-1}$ to $101.8542 \mathrm{mg} \mathrm{L}^{-1}$. This signifies that potassium as the health element for plants helps in the germination process. Humidity, temperature, and moisture are the main growth promoter in this stage. The rate of production of nitrate in the pond has been diminishing by a huge factor of 27.8829 from WAS2 to harvest (Figure 9a) though the fish population in the pond suffice the needs of nitrate as food for the lettuces. The head development stage (WAS2 to WAS5) has an average consumption of 2.1621 $\mathrm{mg} \mathrm{L}^{-1}$ nitrate, $2.2352 \mathrm{mg} \mathrm{L}^{-1}$ phosphate, and $12.9499 \mathrm{mg} \mathrm{L}^{-1}$ potassium. During this stage happens the most significant lettuce growth in terms of canopy area. The white light treatment produced an increase factor of 31.5877 from WAS1 state and red-blue light treatment yielded a factor of 3.33. Phosphate is being consumed on a greater scale from WAS 2 to 3 and WAS4 to 5 (Figure 9b). On the harvesting stage (WAS6), potassium was consumed significantly lower at $8.2321 \%$ as compared to WAS5 (Figure 9c). The chlorophyll concentration on lettuce canopy for both light treatments distinctly intensified from WAS5 to WAS6 (Figure 9d) resulting in a greener leaf surface. It is supported by the increase of 230.39 $\%$ in nitrate consumption in WAS 6 with very low $37.9921 \%$ phosphate and $12.1475 \%$ potassium. Lettuce leaf tissue expands at the average rate of $1.66708 \mathrm{~cm}^{2}$ for white light treatment and $0.23256 \mathrm{~cm}^{2}$ for red-blue light treatment. It is evident that white light promotes better lettuce growth in terms of canopy span area (Figure 9d). Overall, the 40 cultivated lettuces consumed a total of $11.4937 \mathrm{mg} \mathrm{L}^{-1}$ of nitrate, $11.6048 \mathrm{mg} \mathrm{L}^{-1}$ of phosphate, and $78.6155 \mathrm{mg} \mathrm{L}^{-1}$ of potassium through the cultivation cycle from vegetative to harvest stage. The use of the developed wNPK model determined macronutrients present in water sources easier, economical, and efficient. 
A

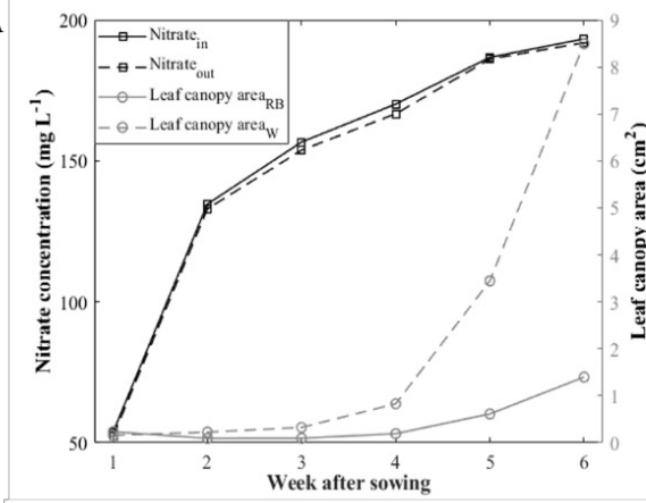

C

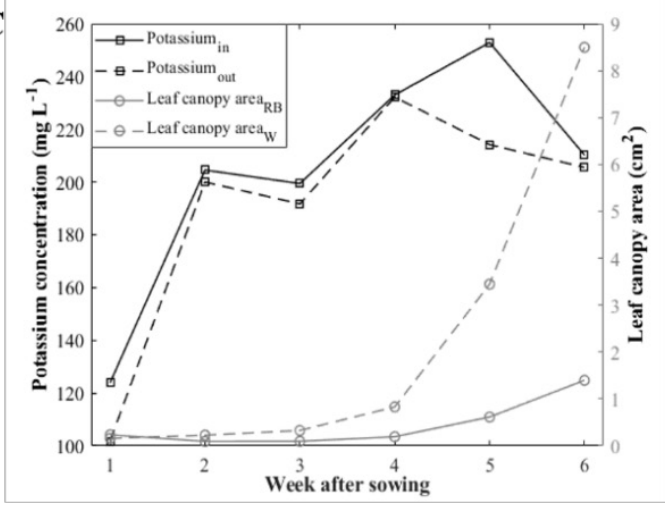

B
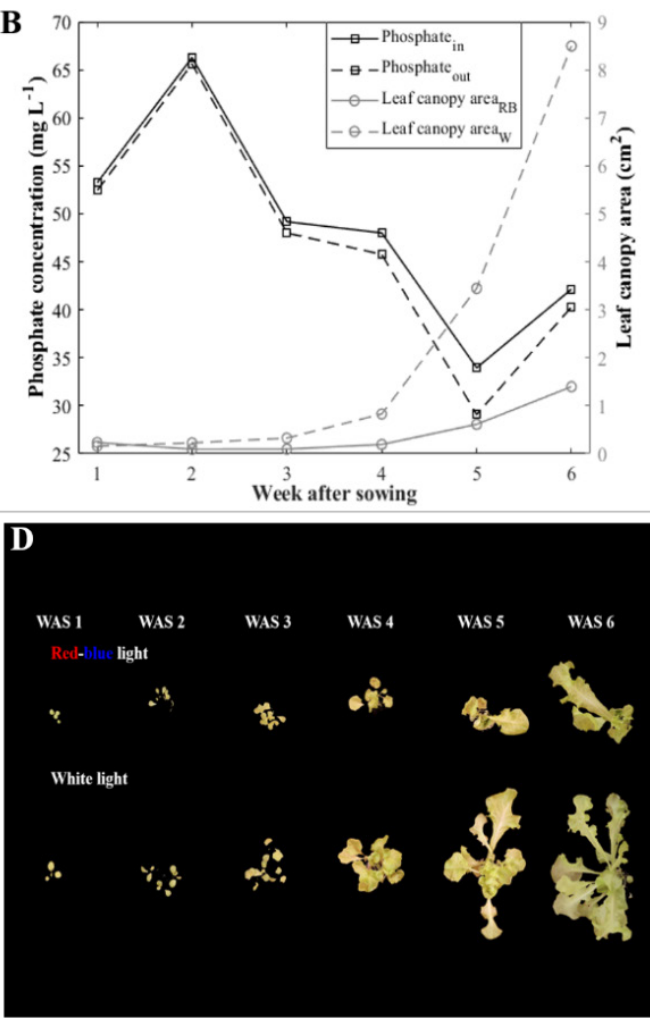

Figure 9 Leaf canopy area response to nutrient uptake in terms of (a) nitrate, (b) phosphate, (c) potassium, and the (d) representative lettuce samples for each light treatment from $1^{\text {st }}$ to $6^{\text {th }}$ week after sowing (WAS)

\section{Conclusions}

Nutrient management system in aquaponic cultivation is of utmost importance for the implementation of computer vision and computational intelligence as planted crops in a controlled environment are dependent on efficient automation for growth promotion. Aquaphotomics analysis confirmed that 205, 840, and $765 \mathrm{~nm}$ wavelengths are the spectral protocol for nitrate, phosphate, and potassium determination in water. The reduction of leaf photosynthetic signatures from 18 to 3 as input predictors to the machine learning model resulted in lower system computational cost. The use of gravitational search algorithm converged to the optimal RNN network architecture resulting in higher sensitivity in predicting macronutrients. The developed wNPK model is a hybrid of neighborhood component analysis, ReliefF, gravitational search, and recurrent neural network that predicts aquaponic pond water macronutrient concentrations in terms of nitrate, phosphate, and potassium based on lettuce leaf canopy photosynthetic signatures through a digital camera. It predicts NPK with 93.61, 84.03 and $91.39 \%$ accuracy, respectively, besting out other feature-based machine learning models such as GPR, SVM, RTree, and TEns. Out of the 3 macronutrients, potassium significantly helps in the germination of seeds and nitrogen plays an important role in intensifying the chlorophyll at the harvest stage. Hence, leaf canopy photosynthetic signatures are a substantial indicator of the existing macronutrient nutrients on its water source. The results in this study pointed out that the use of a single camera to measure both water macronutrient concentrations and crop signature at the same time is an innovative, efficient and economical approach for precision farming. 
http://wjst.wu.ac.th

\section{Acknowledgements}

The authors acknowledge the support of the Engineering Research and Development for Technology (ERDT) of the Department of Science and Technology (DOST) of the Philippines and the Intelligent Systems Laboratory and the Molecular Biology Laboratory of the De La Salle University, Philippines.

\section{References}

[1] A Rawankar, M Nanda, H Jadhav, P Lotekar, R Pawar, L Sibichan and A Pangare. Detection of N, $\mathrm{P}, \mathrm{K}$ fertilizers in agricultural soil with NIR laser absorption technique. In: Proceedings of the $3^{\text {rd }}$ International Conference on Microwave and Photonics, Dhanbad, India. 2018, p. 1-2.

[2] R Concepcion II, S Lauguico, RR Tobias, E Dadios, A Bandala and E Sybingco. Estimation of photosynthetic growth signature at the canopy scale using new genetic algorithm-modified visible band triangular greenness index. In: Proceedings of the IEEE International Conference on Advanced Robotics and Intelligent Systems, Taipei, Taiwan. 2020, p. 1-6.

[3] R Concepcion II, PJ Loresco, RA Bedruz, E Dadios, S Lauguico and E Sybingco. Trophic state assessment using hybrid classification tree-artificial neural network. Int. J. Adv. Intell. Inform. 2020; 6, 46-59.

[4] A Romanelli, DX Soto, I Matiatos, DE Martínez and S Esquius. A biological and nitrate isotopic assessment framework to understand eutrophication in aquatic ecosystems. Sci. Total Environ. 2020; 715, 136909.

[5] S Zhang, W Wang, K Zhang, P Xu and Y Lu. Phosphorus release from cyanobacterial blooms during their decline period in eutrophic Dianchi Lake, China. Enviro. Sci. Pollut. Res. 2018; 25, $13579-88$.

[6] R Concepcion II, J Alejandrino, S Lauguico, RR Tobias, E Sybingco, E Dadios and A Bandala. Lettuce growth stage identification based on phytomorphological variations using coupled color superpixels and multifold watershed transformation. Int. J. Adv. Intell. Inform. 2020; 6, 261-77.

[7] D Neocleous and D Savvas. The effects of phosphorus supply limitation on photosynthesis, biomass production, nutritional quality and mineral nutrition in lettuce grown in a recirculating nutrient solution. Sci. Horticulturae 2019; 252, 379-87.

[8] F Tóth, K Zsuga, E Kerepeczki, L Berzi-Nagy, Z Jakabné Sándor and L Körmöczi. The effect of feed composition on the structure of zooplankton communities in fishponds. Water 2020; 12, 1338.

[9] M Chowdhury, MSN Kabir, HT Kim and SO Chung. Method of pump, pipe, and tank selection for aeroponic nutrient management systems based on crop requirements. J. Agr. Eng. 2020; 51, 119-28.

[10] A Mitra, Pooja and G Saini. Automated Smart Irrigation System (ASIS). In: Proceedings of the International Conference on Computing, Communication and Intelligent Systems, Greater Noida, India. 2019, p. 327-30.

[11] CJ Thirunavuakkarasu, A Bhaskar and A Penujuru. Automated fertigation system for efficient utilization of fertilizer and water. In: Proceedings of the $9^{\text {th }}$ International Conference on Information Technology and Electrical Engineering, Phuket, Thailand. 2017, p. 1-6.

[12] M Mahmud, F Ejeian, S Azadi, M Myers, B Pejcic, R Abbasi and M Asadnia. Recent progress in sensing nitrate, nitrite, phosphate and ammonium in aquatic environment. Chemosphere 2020; 259, 127492.

[13] G Liu, L Deng, R Wu, S Guo, W Du, M Yang and F Chen. Determination of nitrogen and phosphorus fertilisation rates for tobacco based on economic response and nutrient concentrations in local stream water. Agr. Ecosyst. Environ. 2020; 304, 107136.

[14] Y Shi, G Xu, Y Wang, BA Engel, H Peng, W Zhang and M Dai. Modelling hydrology and water quality processes in the Pengxi river basin of the three gorges reservoir using the soil and water assessment tool. Agr. Water Manag. 2017; 182, 24-38. 
http://wjst.wu.ac.th

[15] D Deswati, N Febriani, H Pardi, Y Yusuf and H Suyani. Applications of aquaponics on Pakcoy (Brassica Rapa L) and Nila Fish (Oreochromis Niloticus) to the concentration of ammonia, nitrite, and nitrate. Orient. J. Chem. 2018; 34, 2447-55.

[16] CO Letelier-Gordo, SL Aalto, S Suurnäkki and PB Pedersen. Increased sulfate availability in saline water promotes hydrogen sulfide production in fish organic waste. Aquacult. Eng. 2020; 89, 102062.

[17] K Mendivil-Garcia, LE Amabilis-Sosa, AE Rodríguez-Mata, JG Rangel-Peraza, V GonzalezHuitron and CIG Cedillo-Herrera. Assessment of intensive agriculture on water quality in the Culiacan river basin, Sinaloa, Mexico. Environ. Sci. Pollut. Res. 2020; 27, 28636-48.

[18] R Concepcion II, S Lauguico, J ALejandrino, J de Guia, E Dadios and A Bandala. Aquaphotomics determination of total organic carbon and hydrogen biomarkers on aquaponic pond water and concentration prediction using genetic programming. In: Proceedings of the IEEE Region Ten Humanitarian Technology Conference, Kuching, Malaysia. 2020, p. 1-6.

[19] S Lauguico, R Baldovino, R Concepcion, J Alejandrino, RR Tobias and E Dadios. Adaptive neurofuzzy inference system on aquaphotomics development for aquaponic water nutrient assessments and analyses. In: Proceedings of the $12^{\text {th }}$ International Conference on Information Technology and Electrical Engineering, Yogyakarta, Indonesia. 2020, p. 317-22.

[20] MZ Mokhtar, MS Sofiana, WO Wan Maznah and SY Teh. Water quality assessment of Bukit Merah Reservoir, Malaysia using mathematical modeling. IOP Conf. Ser. Earth Environ. Sci. 2020; $\mathbf{5 3 5}, 012023$.

[21] T Fang, P Li, K Lin, N Chen, Y Jiang, J Chen and J Ma. Simultaneous underway analysis of nitrate and nitrite in estuarine and coastal waters using an automated integrated syringe-pump-based environmental-water analyzer. Anal. Chim. Acta 2019; 1076, 100-9.

[22] T Qi, Q Xiao, Z Cao, M Shen, J Ma, D Liu and H Duan. Satellite estimation of dissolved carbon dioxide concentrations in China's lake Taihu. Environ. Sci. Technol. 2020; 54, 13709-18.

[23] AM Patokar and VV Gohokar. Automatic investigation of micronutrients and fertilizer dispense system using microcontroller. In: Proceedings of the International Conference on Recent Innovations in Electrical, Electronics \& Communication Engineering, Bhubaneswar, India. 2018, p. 1737-9.

[24] A Gupta, N Sharma and H Sharma. Fitness based gravitational search algorithm. In: Proceedings of the International Conference on Computing, Communication and Automation, Greater Noida, India. 2016, p. 309-14.

[25] $\mathrm{H} \mathrm{Hu}, \mathrm{X}$ Cui and Y Bai. Two kinds of classifications based on improved gravitational search algorithm and particle swarm optimization algorithm. Adv. Math. Phys. 2017; 2017, 1-7.

[26] E Rashedi, H Nezamabadi-pour and S Saryazdi. GSA: A gravitational search algorithm. Inform. Sci. 2009; 179, 2232-48.

[27] PJM Loresco, IC Valenzuela and EP Dadios. Color space analysis using KNN for lettuce crop stages identification in smart farm setup. In: Proceedings of the IEEE Region 10 International Conference TENCON. Jeju, South Korea. 2018, p. 2040-4.

[28] S Alberto, DSJ Carlos, DF Pedro, EBB Marcos, FS Lafayette and LDSF Rener. Chlorophyll and macronutrients content in leaf tissue of Musa sp Prata-An under fertigation. Afr. J. Agr. Res. 2014; 9, 1714-20.

[29] AC Edwards, PS Hooda and Y Cook. Determination of nitrate in water containing dissolved organic carbon by ultraviolet spectroscopy. Int. J. Environ. Anal. Chem. 2001; 80; 49-59.

[30] YK Mahadevaiah, MS Abdul Galil, MS Suresha, MA Sathish and G Nagendrappa. A simple spectrophotometric determination of phosphate in sugarcane juices, water and detergent samples. $E$. J. Chem. 2007; 4, 467-73.

[32] XY Jian, XQ Chen and M Xu. Rapid determination of trace potassium in drinks and serum by rayleigh light scattering technique. J. Food Drug Anal. 2007; 15, 178-84. 